P4-368

\section{PRE-STATISTICAL HARMONIZATION OF COGNITIVE MEASURES ACROSS SIX POPULATION-BASED COHORTS: ARIC, CARDIA, CHS, FHS, MESA, AND NOMAS}

Emily M. Briceño ${ }^{1}$, Alden L. Gross ${ }^{2}$, Bruno Giordani ${ }^{3}$, Jennifer J. Manly ${ }^{4}$, Rebecca F. Gottesman ${ }^{5}$, Mitchell S. V. Elkind ${ }^{4}$, Stephen Sidney ${ }^{6}$, Stephanie Hingtgen ${ }^{1}$, Deborah Levine ${ }^{1},{ }^{1}$ University of Michigan, Ann Arbor, MI, USA; ${ }^{2}$ Johns Hopkins Bloomberg School of Public Health, Baltimore, MD, USA, ${ }^{3}$ Michigan Alzheimer's Disease Center, Ann Arbor, MI, USA; ${ }^{4}$ Columbia University, New York, NY, USA; ${ }^{5}$ Johns Hopkins University, Baltimore, MD, USA; ${ }^{6}$ Kaiser Permanente Division of Research, Oakland,CA, USA. Contact e-mail: emilande@med.umich.edu

Background: To study the association between blood pressure levels over the life course and cognitive impairment and dementia risk in Blacks, Whites, and Hispanics, we aimed to harmonize cognitive instruments across six longitudinal population-based cohorts: the Coronary Artery Risk Development in Young Adults study (CARDIA), the Northern Manhattan Study (NOMAS), the Atherosclerosis Risk in Communities (ARIC) study, the Cardiovascular Health (CHS) study, the Multi-Ethnic Study of Atherosclerosis (MESA) study, and the Fra- mingham Offspring Study (FOS). Methods: We identified cognitive instruments across the $6 \mathrm{NIH}$-funded cohort studies, representing tests of global cognition, language, executive functioning, and memory. As part of pre-statistical harmonization prior to psychometric harmonization using item response theory methods, we conducted an extensive review of the administration, scoring procedures, and score ranges of each cognitive test in each study to ensure comparability of instrument scores across studies. Results: Animal naming spanned all six studies, letter fluency spanned five studies, and four instruments (Mini Mental State Examination, Boston naming, digit span, and digit symbol substitution) spanned four studies (Table). A detailed review of administration and scoring procedures revealed variability in format, content, administration, and scoring procedures, even for seemingly equivalent tests based on instrument titles and general descriptions. For example, animal naming procedures differed from naming four-legged animals within a 30-second time limit (two studies) to naming any animals within a 60 -second time limit (four studies). In other instruments, differences were found across cohorts with regard to test version, specificity of scoring guidelines, and number of items. These procedural differences led to variability across

Table

Cognitive Instruments of Cohort Studies. Related instruments highlighted in maize

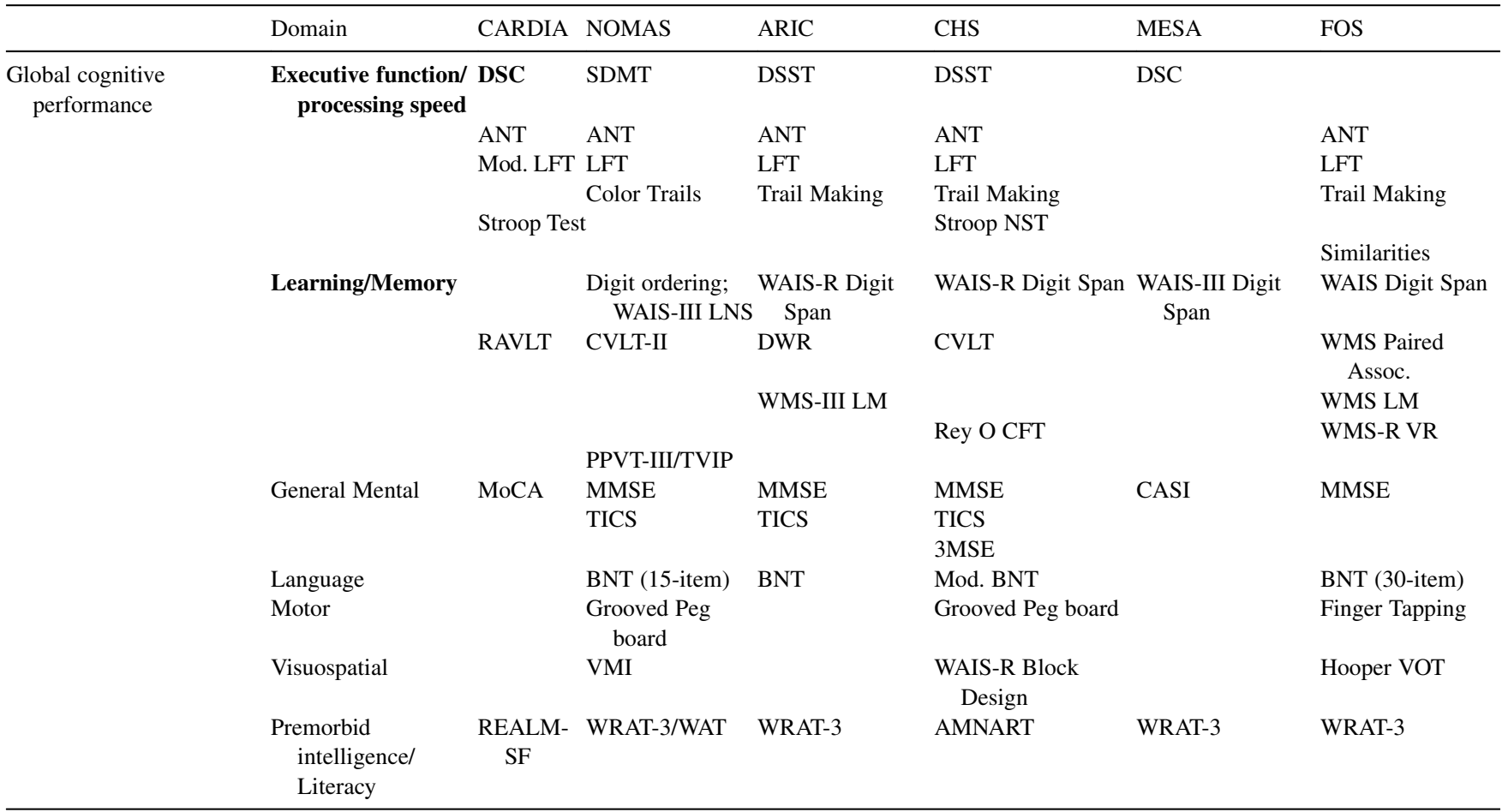

Note: Cognitive instruments were administered to different participant samples and at different time intervals across the 6 cohort studies.

Abbreviations: Cohort studies: CARDIA is the Coronary Artery Risk Development in Young Adults study. NOMAS is the Northern Manhattan Study. ARIC is the Atherosclerosis Risk in Communities Study. CHS is the Cardiovascular Health Study. MESA is the Multi-Ethnic Study of Atherosclerosis study. FOS is the Framingham Offspring Study.

Cognitive Instruments: 3MSE is the Modified Mini Mental State Examination. AMNART is the American Version of the National Adult Reading Test. ANT is Animal Naming Test. BNT is Boston Naming Test. CASI is Cognitive Abilities Screening Instrument. CVLT is California Verbal Learning Test. DSC is WAISIII Digit Symbol Coding Test. DSST is the WAIS-R Digit Symbol Substitution Test. DWR is the Delayed Word Recall test. Hooper VOT is Hooper Visual Organization Test. LFT is Letter Fluency Test. LM is Logical Memory. LNS is Letter-Number Sequencing. MMSE is Mini-Mental State Examination. MoCA is Montreal Cognitive Assessment. PPVT is Peabody Picture Vocabulary Test. RAVLT is Rey Auditory Verbal Learning Test (RAVLT). REALM-SF is Rapid Estimate of Adult Literacy in Medicine. Rey O CFT is Rey-Osterrieth Complex Figure Test. SDMT is Symbol Digit Modalities Test. Similarities is the WAIS Similarities test. Stroop NST is the Stroop Neuropsychological Screening Test. TICS is the Telephone Interview for Cognitive Status. TVIP is Test de Vocabulario en Imagenes Peabody, Hispanic-American Adaptation. WAT is the Word Accentuation Test. WRAT is the Wide Range Achievement Test. WRAT-3 is Wide Range Achievement Test, Third Edition Reading tests. VMI is Developmental Test of Visuomotor Integration. VR is Visual Reproduction. WAIS is Wechsler Adult Intelligence Scale. WAIS-R is Wechsler Adult Intelligence Scale-Revised. WAIS-III is Wechsler Adult Intelligence Scale-3rd Edition. WMS is Wechsler Memory Scale. WMS-R is WMS-Revised. WMS-III is WMS-3rd Edition. 
cohorts in individual score ranges (e.g., from 0-10 for the 30-second animal naming version to 1-39 for the 60-secnd version). Conclusions: Harmonization of cognitive instruments requires careful scrutiny of test questions and score ranges to determine degree of test equivalence and use of appropriate statistical strategies to account for differences. Pre-statistical harmonization performed here will facilitate subsequent approaches to formally harmonize cognitive performance at the level of constructs by leveraging assorted methods based in item response theory and percentile equating.

\section{P4-369 PREVALENCE OF COGNITIVE AND VESTIBULAR IMPAIRMENT IN COMMUNITY FALL PREVENTION PROGRAMS}

Brenda Maricela Varriano ${ }^{1}$, Shaleen Sulway ${ }^{2}$, Curt Wetmore ${ }^{2}$, Wanda WA. Dillon ${ }^{2}$, John Rutka ${ }^{2}$, Maria Carmela Tartaglia ${ }^{3},{ }^{1}$ Tanz Centre for Neurodegenerative Disease, University of Toronto, Toronto, ON, Canada; ${ }^{2}$ Hertz Multidisciplinary Neurotology Clinic, Toronto General Hospital, Toronto, ON, Canada; ${ }^{3}$ Tanz Centre for Research in Neurodegenerative Diseases, University of Toronto, Toronto, ON, Canada. Contacte-mail: brenda.varriano@mail.utoronto.ca

Background: Falls are a growing concern in seniors ( $>65$ years old). Cognitive impairment (CI) and vestibular impairment (VI) increases the risk of falls. Fall prevention programs (FPPs) are used to prevent falls, however fall prevalence continues to increase. We assessed the prevalence of CI and VI in community dwelling seniors attending FPPs. Methods: Patients ( $>65$ years old) were recruited from two FPPs. All patients fell in the past year. CI was assessed using the Montreal Cognitive Assessment (MoCA). MoCA $<26$ indicated CI. Definite vestibular impairment (DVI) was diagnosed if failed one of the following tests: Head Impulse- (video and bedside), Head Shake- or Dix Hallpike test. Probable Vestibular Impairment

Table 1

Site Demographics of Fall Prevention Programs

\begin{tabular}{lccc}
\hline & & Care First & $\begin{array}{c}\text { Circle of } \\
\text { Care }(\mathrm{N}=17)\end{array}$ \\
\hline Age & Total $(\mathrm{N}=29)$ & Seniors $(\mathrm{N}=12)$ & $83.0 \pm 6.5$ \\
Gender M:F (\%) & $81.2 \pm 7.1$ & $79.0 \pm 7.6$ & $4: 13(23: 77)$ \\
Prevalence DVI & $9: 20(31: 69)$ & $11 / 12(91.7 \%)$ & $15 / 17(88.2 \%)$ \\
Prevalence CI & $26 / 29(89.7 \%)$ & $8 / 12(75.0 \%)$ & $14 / 17(82.4 .2 \%)$ \\
Both DVI and CI & $22 / 29(75.9 \%)$ & $7 / 12(58.3 \%)$ & $12 / 17(76.5 \%)$ \\
Both pVI and CI & $19 / 29(69.0 \%)$ & $1 / 12(8.3 \%)$ & $1 / 17(5.9 \%)$ \\
\hline
\end{tabular}

CI-Cognitive Impairment, DVI-Definite Vestibular Impairment, M:F - male to female ratio, pVI-Probable Vestibular Impairment

Table 2

Abnormal Tests of Vestibular Functioning at Fall Prevention Programs $(\mathrm{N}=28)$

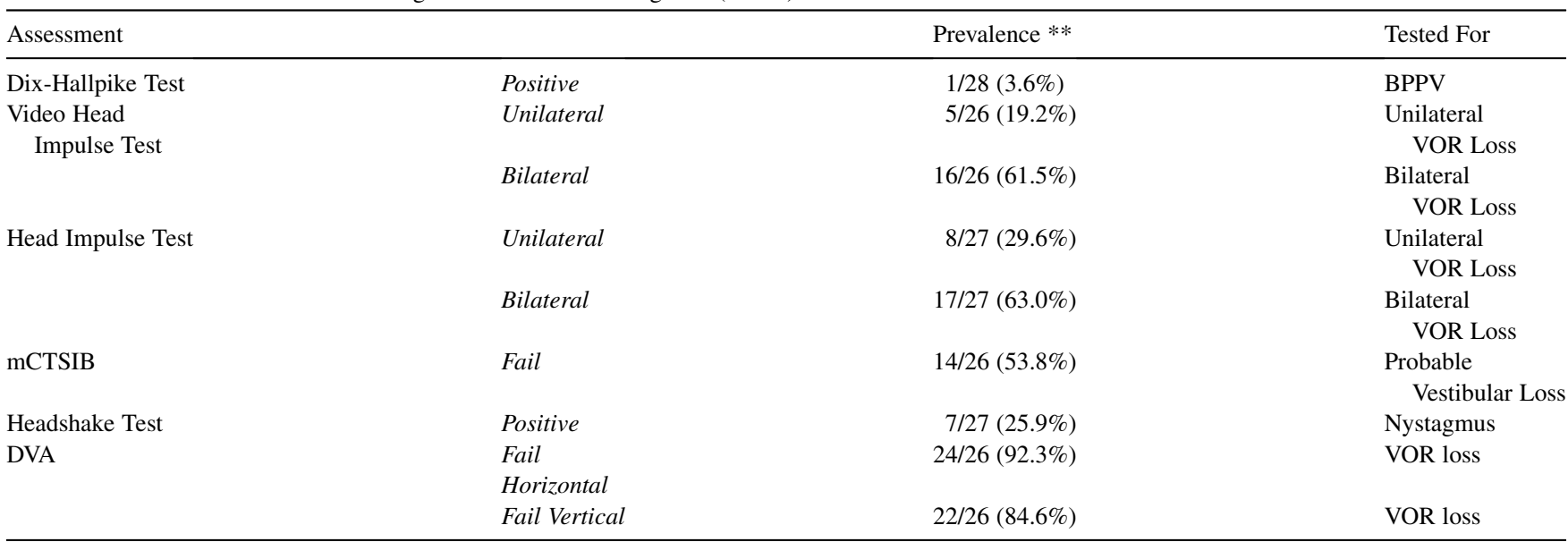

BPPV - Benign paroxysmal positional vertigo, DVA- dynamic visual acuity, VOR - Vestibulo-ocular reflex

Table 3

Prevalence of vestibular impairment and questionnaire results at Fall Prevention Programs $(\mathrm{N}=29)$

\begin{tabular}{|c|c|c|c|c|c|}
\hline & Total DM & $D V I-U V H$ & $D V I-B V H$ & $\mathrm{pVI}$ & NVI \\
\hline Age $\pm S D$ & $81.3 \pm 7.3$ & $79.3 \pm 9.0$ & $81.8 \pm 7.1$ & $83.5 \pm 2.1$ & $78 \pm 0.0$ \\
\hline Lines lost DVA $\pm \mathrm{SD}$ (vertical) & $2.8 \pm 1.4$ & $2.3 \pm 1.2$ & $2.9 \pm 1.5$ & $3.0 \pm 0.0$ & $2.0 \pm 0.0$ \\
\hline $\mathrm{ABC}(\%)$ & $61.6 \pm 27.0$ & $51.9 \pm 24.4$ & $65.0 \pm 27.7$ & $68.1 \pm 27.4$ & $100 \pm 0.0$ \\
\hline DHI $(/ 100)$ & $23.8 \pm 21.6$ & $30 \pm 27.1$ & $21.9 \pm 20.0$ & $26 \pm 25.5$ & $0.0 \pm 0.0$ \\
\hline
\end{tabular}

ABC-activities specific balance confidence scale, BVH - Bilateral vestibular hypofunction DHI-dizziness handicap inventory, DVI - Definite Vestibular Impairment, MoCA-Montreal cognitive assessment, NVI- no vestibular impairment, pVI - probable vestibular impairment, UVH - Unilateral vestibular hypofunction 